\title{
La relève dans les PME manufacturières québécoises : un portrait de la MRC de Drummond
}

\author{
Louise Cadieux, DBA et \\ Brigitte Prud'homme \\ Université du Québec à Trois-Rivières
}

\section{Introduction}

L'économie québécoise, comme celle de nombreuses régions industrialisées, repose en très grande partie sur les PME dont le tiers sont situées dans des petites villes et villages et environ le quart dans les banlieues et les centres urbains ou les centres-villes respectivement ${ }^{1}$. Réelle pépinière de PME, la MRC de Drummond compte 507 entreprises manufacturières lesquelles, selon une estimation de 2005, procuraient un emploi à 16023 personnes. De ces entreprises, $92 \%$ comptaient moins de 100 employés, tandis que $8 \%$ en employaient plus de 100 . Ayant été pour la majorité fondées après les années 1960, plusieurs de ces entreprises vitalisant le dynamisme de la MRC de Drummond sont, comme une très grande majorité de nos en-

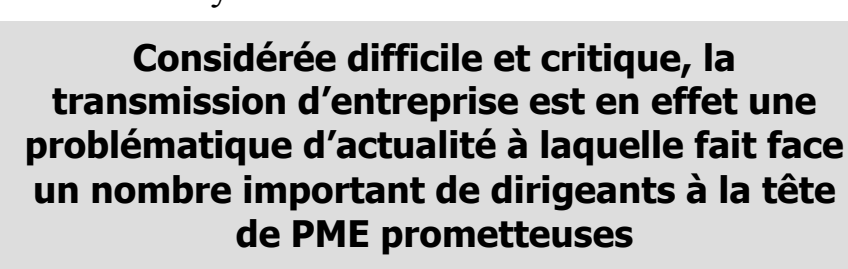

Considérée difficile et critique, la treprises québécoises et canadiennes, arrivées à un nouveau tournant de leur histoire. Considérée difficile et critique, la transmission d'entreprise est en effet une problématique d'actualité à laquelle fait face un nombre important de dirigeants à la tête de PME prometteuses, notamment pour l'emploi et le tissu économique de plusieurs pays industrialisés. A ce sujet, les résultats d'une enquête de la Fédération canadienne de l'entreprise indépendante (FCEI), dévoilés en 2005, montrent que $71 \%$ des dirigeants de PME en poste prévoient procéder au transfert de leur entreprise au cours des 10 prochaines années et $41 \%$ dans 5 ans $^{2}$.

Selon la FCEI et OSEO ${ }^{3}$, une transmission d'entreprise réussie présente des avantages économiques pouvant aller au-delà du maintien des emplois et de la production actuelle. Pour ces organis- mes, les repreneurs «qu'ils soient familiaux ou non » apportent une nouvelle vigueur aux entreprises existantes ainsi que de meilleures occasions pour les employés, leur collectivité et l'économie. Toutefois, l'aventure s'avère risquée. Par exemple, dans le cadre d'une étude longitudinale faite auprès de plus de 3000 PME françaises ayant vécu l'expérience d'une transmission, les résultats du rapport déposé par OSEO montrent qu'en moyenne, le taux de défaillance s'établit à $5 \%$ après deux ans d'opération, à $13,5 \%$ après quatre ans et à $21 \%$ après six ans. C'est, par conséquent, dans cette perspective que nous avons réalisé une étude ayant pour objectif de dresser un portrait, le plus précis possible, de l'état de la situation de la relève dans la MRC de Drummond. Pour les parties prenantes impliquées dans le projet, les résultats permettront d'élaborer de nouvelles stratégies d'intervention adaptées aux réalités des propriétaires-dirigeants des PME manufacturières faisant face à la transmission de leur entreprise et ainsi, pérenniser le dynamisme d'une région connue pour son avant-gardisme en matière de développement économique.

\section{La cueillette de données}

Pour connaître la situation de la relève dans la MRC de Drummond, un questionnaire a été posté à 410 propriétaires-dirigeants de PME manufacturières y œuvrant. Comme le montre le tableau 1, parmi les 135 entreprises répondantes, 128 ont été retenues pour fins d'analyse, pour un taux de réponse 
de $31 \%$. Parmi celles-ci, $41 \%$ sont des entreprises « artisans », les «petites » entreprises représentent $39 \%$ de l'échantillon final, tandis que les «moyennes » entreprises comptent pour $20 \%$.

Comparée aux autres enquêtes sur la relève, notre étude se distingue. D'abord, bien que l'information sur les participants soit confidentielle, la collecte de données ne s'est pas faite de façon anonyme. Cela nous a, entre autres, permis de contacter les propriétaires-dirigeants lorsque nous avions besoin d'éclaircissements. Ce qui s'est produit pour une grande partie des répondants et qui explique le si faible taux de rejet des questionnaires reçus pour fins d'analyse. Ensuite, ce sont les propriétairesdirigeants eux-mêmes qui ont répondu au questionnaire, ce qui est rarement le cas des études faites sur le sujet. Enfin, notre étude se distingue aussi par l'attention que nous avons apportée aux trois catégories de PME visées par les intervenants socioéconomiques de la MRC de Drummond, soit les « artisans », les « petites » et les « moyennes », à la diversité des parcours de chacune des PME manufacturières participantes, ainsi qu'à l'attitude des propriétaires-dirigeants face à la relève.

Tableau 1

Taux de réponse réparti en fonction de la taille des PME participantes

\begin{tabular}{|l|c|c|c|c|c|c|}
\hline & \multicolumn{9}{c|}{ Questionnaires } \\
\hline & Postés & \multicolumn{2}{c|}{ Reçus } & Rejetés & \multicolumn{2}{c|}{ Taux de réponse final } \\
\hline Artisans (1-5es) & 201 & 57 & $28 \%$ & 5 & 52 & $26 \%$ \\
\hline Petites (6-25es) & 138 & 52 & $38 \%$ & 1 & 51 & $37 \%$ \\
\hline Moyennes (26-100es) & 71 & 26 & $37 \%$ & 1 & 25 & $35 \%$ \\
\hline Total & $\mathbf{4 1 0}$ & $\mathbf{1 3 5}$ & $\mathbf{3 2 , 9 \%}$ & $\mathbf{7}$ & $\mathbf{1 2 8}$ & $\mathbf{3 1 \%}$ \\
\hline
\end{tabular}

\section{Les résultats de l'étude}

Selon les experts en la matière, la première expérience en transmission d'entreprise est critique. Avant d'entrer dans le cœur du sujet, nous avons donc voulu connaître ce qu'il en était des PME manufacturières de la MRC de Drummond. À ce sujet, et comme le montre la figure 1, parmi les PME répondantes, nos résultats révèlent qu'au moment de l'enquête, $59 \%$ d'entre elles étaient de première génération, donc n'avaient aucune expérience de transmission; alors que $14 \%$ avaient vécu au moins un transfert générationnel. Aussi, notons que $12 \%$ des PME répondantes étaient dirigées et contrôlées par deux générations, ce qui signifie qu'elles étaient en cours de processus de transmission tandis que $15 \%$ étaient des cas de « reprises », ce qui indique qu'elles avaient à leur actif une expérience de transmission, autre que familiale.

Ce qu'il faut retenir de ces premiers résultats est la proportion élevée de PME manufacturières qui sont de $1^{\text {re }}$ génération, soit $59 \%$, dont $84 \%$ sont des entreprises procurant de l'emploi à un maximum de 25 personnes. À ce chapitre, excluant les entreprises où il $\mathrm{y}$ a deux générations en poste, les résultats relatifs aux PME manufacturières de la MRC de Drummond sont un peu plus élevés que ceux obtenus par la FCEI, lesquels révélaient que $53 \%$ des PME canadiennes sondées appartenaient toujours à leur fondateur ou étaient exploitées par lui. $\mathrm{Au}$ reste, les résultats compilés montrent que $100 \%$ des PME de $1^{\text {re }}$ génération ont été fondées après 1960 . Parmi elles, $13 \%$ l'ont été entre 1960 et 1979, $28 \%$ entre 1980 et 1989 et $59 \%$ après 1990. En bref, considérant que les entreprises ayant été fondées avant 1990 sont les plus susceptibles d'être préoccupées par leur pérennité, il nous est possible d'avancer avec assurance qu'au minimum $41 \%$ des entreprises de $1^{\text {re }}$ génération ayant participé à notre étude seront rapidement concernées par la problématique de leur première transmission, et ce, quelle qu'en soit la nature et que $68 \%$ des entreprises ayant été « reprises » le seront tout autant. 


\section{Figure 1 \\ Le profil des expériences de transmission des PME manufacturières participantes}

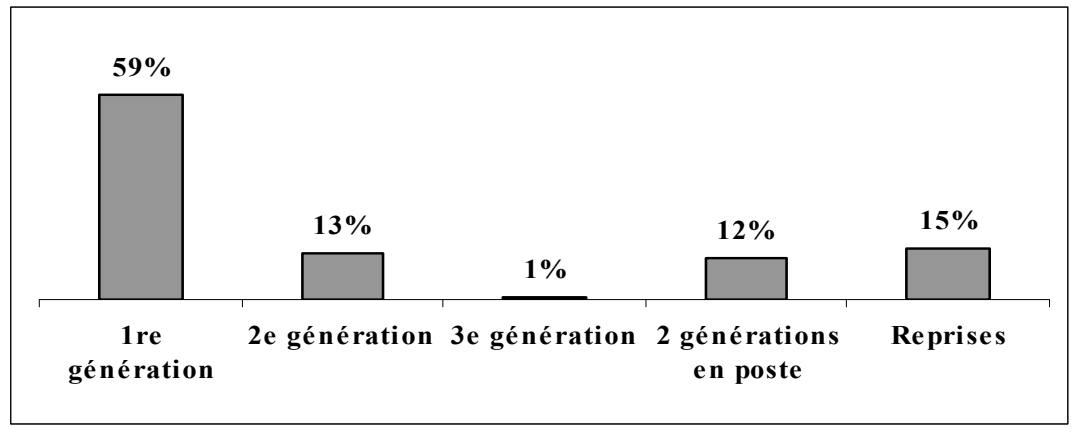

Selon OSEO, « le départ à la retraite de l'ancien dirigeant est le premier motif de transmission. Cette évolution est liée à la pyramide des âges des dirigeants. » Voilà pourquoi parmi les préoccupations des partenaires impliqués dans le projet, les questions relatives aux propriétaires-dirigeants étaient importantes, entre autres, en ce qui concerne leur âge, leur implication dans l'entreprise, leurs perspectives de retraite et leurs préoccupations face à la relève. Voici ce que nous avons appris.

D'abord, en ce qui concerne l'âge, notons que $57 \%$ des propriétaires-dirigeants des PME participantes ont 45 ans et plus. Parmi eux, $44 \%$ sont à la tête d'entreprises « artisans », $40 \%$ aux commandes de «petites» entreprises et $16 \%$ dirigent une «moyenne» entreprise. En bref, $84 \%$ des participants âgés de 45 ans et plus dirigent une PME manufacturière procurant de l'emploi à un maximum de 25 personnes, ce qui laisse supposer l'existence d'un minimum de planification formelle dans l'ensemble de leurs processus de gestion, notamment en ce qui concerne la relève. À ce sujet, notons que selon la FCEI, seuls $35 \%$ des répondants avaient un plan pour vendre ou transmettre l'entreprise ou réduire leurs activités futures et que ce plan était plus souvent non écrit ou informel, ce qui, nous devons l'admettre est assez représentatif des comportements de planification des dirigeants de PME. Cette absence de planification familière aux PME, surtout dans les plus petites, est-elle nécessairement présage d'échec? A ce sujet, il est impossible de se prononcer puisque, à ce jour, le lien entre les activités de planification et la réussite de la transmission de l'entreprise n'est pas encore prouvé, cela même si plusieurs chercheurs s'y intéressent depuis plusieurs années.

\section{$57 \%$ des propriétaires-dirigeants des PME participantes ont $\mathbf{4 5}$ ans et plus.}

Dans un processus de réflexion sur la relève, il est important de connaître l'implication des propriétaires-dirigeants dans leur entreprise. Plus ceux-ci sont impliqués dans différentes activités, plus il leur sera difficile de quitter leur entreprise. Questionnés à ce sujet, nos résultats montrent que $89 \%$ des répondants sont impliqués dans toutes les sphères d'activité de leur entreprise, qu'elles soient opérationnelles ou stratégiques. Bien que cette proportion soit courante dans les PME, lorsqu'il s'agit d'assurer la pérennité de son entreprise, cela peut aussi être un indice de l'état d'avancement de la relève. Les principales difficultés liées à la trop grande implication des dirigeants dans leur entreprise pourraient, en ce qui nous concerne, engendrer deux principales difficultés. La première concerne l'incapacité à lâcher prise des propriétairesdirigeants due, entre autres, au peu de centres d'intérêts diversifiés tant dans leur vie personnelle, familiale que sociale, que les nombreuses heures de travail n'ont pas permis de développer. Conséquemment, moins ils ont d'intérêts à l'extérieur de leurs activités d'affaires, plus ils sont susceptibles de retarder la mise en œuvre du projet de relève. Quant à la seconde difficulté, elle touche plus précisément le transfert du savoir-faire, souvent essentiel pour la bonne marche de l'entreprise. Plus les propriétaires-dirigeants sont impliqués dans un grand nombre d'activités dans leur entreprise, moins ils ont de chance d'avoir transféré les connaissances qu'ils ont acquises au fil des ans avant l'arrivée officielle du candidat à la relève. 
Les perspectives d'avenir des propriétairesdirigeants en poste sont des points d'informations déterminants auxquels nous devons porter attention lorsqu'il est question de relève. Contrairement à ce qui a été fait dans d'autres études, pour connaître les intentions des propriétaires-dirigeants à l'égard de leur retrait de l'entreprise, nous avons posé deux séries de questions. La première porte sur le transfert de la direction et la seconde sur le transfert de la propriété. Cette préoccupation à double volet s'explique principalement par un constat commun de la part des experts en matière de transmission d'entreprise. Bien que le transfert de la propriété n'évolue pas au même rythme que celui de la direction et que leurs dynamiques respectives aient leur propre lot de particularités, étant ensemble essentiels, les deux types de transferts doivent être pris en compte dans le processus de la transmission d'une entreprise.

\section{Les propriétaires-dirigeants ont une meil- leure idée de leur perspective d'avenir lors- qu'il s'agit de se retirer de la direction de leur entreprise qu'en ce qui concerne leur retrait du capital-actions de ces mêmes entreprises.}

D'abord, à la question « Quand prévoyez-vous vous retirer de la direction de votre entreprise? », nos résultats révèlent que $24 \%$ des répondants prévoient passer à l'acte d'ici 5 ans; $19 \%$ dans 10 ans; tandis que $57 \%$ disent ne pas savoir quand ils se retireront de la direction de leur entreprise, ce qui est considérable si l'on compare nos résultats à d'autres études faites sur le sujet ${ }^{4}$. Maintenant en ce qui a trait à la question «Quand prévoyez-vous avoir procédé au transfert complet de la propriété de votre entreprise? », nos résultats montrent que $19 \%$ des répondants estiment le faire dans 5 ans; $11 \%$ dans 10 ans; tandis que $70 \%$ des répondants ne savent pas quand ils auront procédé au transfert complet de la propriété de leur entreprise. En somme, lorsque questionnés séparément sur les deux volets de la transmission, les propriétairesdirigeants ont une meilleure idée de leur perspective d'avenir lorsqu'il s'agit de se retirer de la direction de leur entreprise qu'en ce qui concerne leur retrait du capital-actions de ces mêmes entreprises. Aussi surprenant que cela puisse paraître, cela correspond néanmoins à la tendance lourde observée depuis quelques années à l'effet que les dirigeants d'entreprises de toutes tailles restent impliqués dans la propriété de leur entreprise, cela même après en avoir transmis la gouvernance. Selon la littérature consultée, deux phénomènes pourraient expliquer cette tendance. Le premier concerne la très forte propension des dirigeants à investir tous leurs avoirs dans leur entreprise et à vouloir garder le même rythme de vie après en avoir transmis la gouvernance. Quant au second phénomène, il s'agit de la possibilité que les dirigeants, à titre de président du conseil, demeurent longtemps en contrôle du capital-actions de l'entreprise, surtout si elle reste familiale.

Les résultats au sujet des horizons en termes d'années des dirigeants des PME manufacturières de la MRC de Drummond laissent songeur, surtout en ce qui concerne ceux qui sont âgés de plus de 45 ans et qui n'ont pas encore déterminé, dans combien de temps ils prévoient se retirer de leur entreprise. Ce qui représente un peu plus de la moitié des répondants $(55 \%$ pour le transfert de la direction et $53 \%$ pour le transfert de la propriété). Pourtant, à ce sujet, tous les experts dans le domaine de la transmission d'entreprise s'entendent. Dépendant du type de la transmission prévue (familiale, interne, externe ou mixte), il faut entre deux et dix ans avant que les candidats à la relève deviennent autonomes et, surtout, efficients. Quant au transfert de la propriété, dans les meilleures conditions, il faudrait entre 9 et 12 mois avant que la transaction arrive à terme ${ }^{5}$, et cela exclut la période de réflexion précédant la mise en œuvre du projet de relève qu'il s'agisse du transfert de la direction ou de la propriété.

Par ailleurs, pour connaître les sources de résistance face à la relève, les propriétaires-dirigeants ont été invités à indiquer, sur une échelle de 1 (pas du tout important) à 5 (très important) si certains énoncés constituaient une préoccupation à l'égard de la relève. Nos résultats révèlent que les propriétairesdirigeants interrogés seraient plus préoccupés par des facteurs concernant «la valeur» de leur entreprise que par des facteurs « familiaux », comme la préservation de l'harmonie familiale et la conservation du patrimoine familial. En bref, et comme l'illustre la figure 2, si protéger la réputation de l'entreprise est le plus important pour eux, obtenir un bon prix pour leur entreprise peut équivaloir à ce 
qu'ils attendent d'une retraite confortable. A ce chapitre, les résultats de la FCEI sont clairs : « $90 \%$ des propriétaires de petites entreprises interrogés ont indiqué qu'ils considéraient le produit de la vente de leur entreprise, leurs épargnes personnelles et l'exonération cumulative de $500000 \$$ des gains en capital comme très importants pour le financement de leur retraite $»$. En bref, à la lumière de nos résultats, les propriétaires-dirigeants dans la MRC de Drummond auraient le même comportement que bien d'autres en termes de préparation à la retraite. En fait, la situation à laquelle ils sont confrontés est préoccupante puisque ce sont habituellement les revenus générés par l'entreprise qui leur a permis d'une part, de subvenir à leurs besoins et à ceux des membres de leurs familles et, d'autre part, à leur procurer la qualité de vie à laquelle ils sont habitués.

\section{Obtenir un bon prix pour leur entreprise peut équivaloir à ce qu'ils attendent d'une retraite confortable.}

Figure 2

Les préoccupations prioritaires des propriétaires-dirigeants à l'égard de la relève

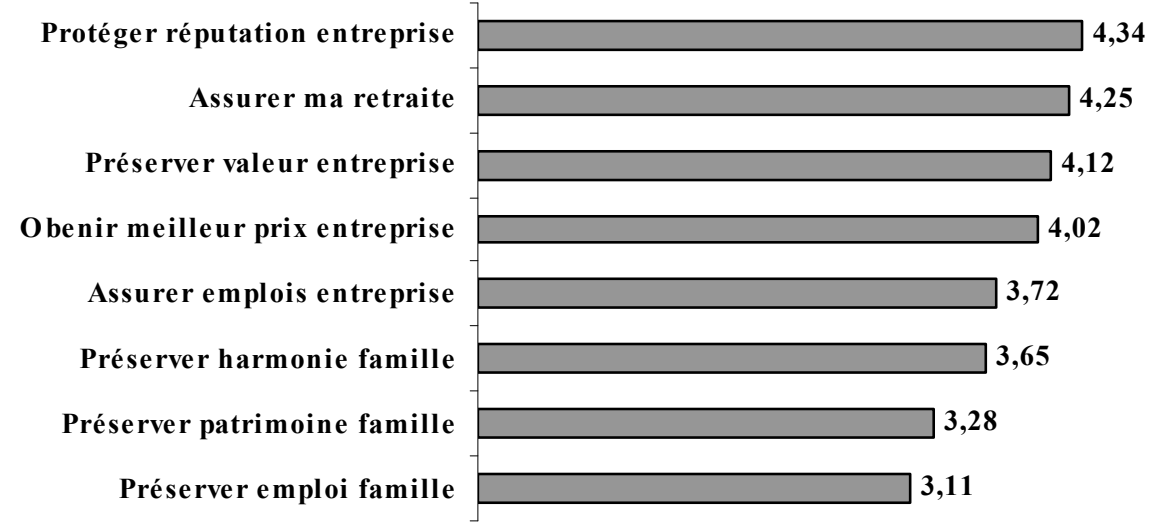

Dans un second temps, et tel que présenté à la figure 3 , les résultats révèlent que trouver une "relève compétente » est la plus importante difficulté pour les propriétaires-dirigeants des PME participantes [classé de 1 (très facile) à 5 (très difficile)]. Ce point est d'ailleurs lié au précédent en ce sens que la réputation de l'entreprise est plus facile à protéger lorsque la relève est assurée par un successeur compétent. Or, cet aspect de la dynamique de la transmission est important, notamment en raison de ré- sultats d'études montrant dans quelle mesure la présence d'un successeur compétent serait garante de la mise en place d'activités de planification de la part du propriétaire-dirigeant ${ }^{6}$. Certains avancent même que les entreprises ayant le mieux réussi sont celles où les dirigeants ont pris le temps de choisir leurs successeurs et n'ont rien fait d'officiel tant et aussi longtemps que la relation existant entre les deux ne soit à leur satisfaction.

Figure 3

\section{Les difficultés des propriétaires-dirigeants à l'égard de la relève}

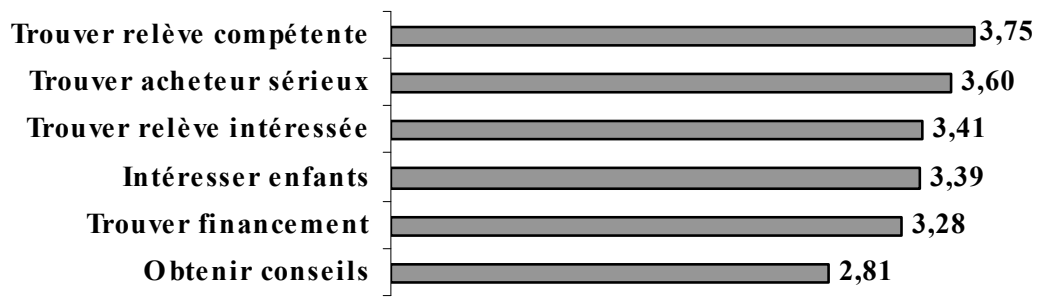


Parmi les difficultés rencontrées par les propriétaires-dirigeants des PME participantes, notons que l'accès au financement arrive pratiquement bonne dernière alors que selon la FCEI, cela est le principal obstacle à la planification de la relève. En revanche, nos résultats montrent que, lorsque analysées distinctement, les préoccupations des dirigeants de PME seraient plus importantes lorsqu'il s'agit du transfert de la direction que celui de la propriété. Par conséquent, bien que le transfert de la propriété et le transfert de la direction soient indispensables au bon déroulement de la transmission d'une entreprise, les considérer séparément peut faciliter l'élaboration de stratégies cohérentes lors d'une telle démarche.

\section{Pour la majorité des propriétaires- dirigeants des PME manufacturières de la MRC de Drummond, assurer la relève de leur entreprise est important ou très impor- tant.}

Enfin, lorsque la relève est à l'ordre du jour, il est nécessaire de faire une distinction entre les PME qui seront transmises à la famille et les autres, notamment pour nous permettre, encore une fois, d'évaluer le niveau de risque. À titre d'exemple, l'étude d'OSEO révèle que « le niveau de risque est quasi nul pour les transmissions familiales, et un ancien salarié de l'entreprise a deux fois plus de chances de réussite qu'un repreneur externe ». Questionnés à cet effet, nos résultats, révèlent que pour $26 \%$ des propriétaires-dirigeants des PME répondantes, la famille est une relève sérieusement envisagée tandis que $34 \%$ attendent que leurs enfants soient en âge de prendre une telle décision avant de se prononcer, ce qui, pour nous, est une attitude d'ouverture favorable à l'égard de la relève familiale. Au reste, notons que pour $41 \%$ des propriétaires-dirigeants des PME manufacturières de la MRC de Drummond, il serait trop tôt pour penser à la relève. Parmi eux, $65 \%$ ont moins de 45 ans tandis que $35 \%$ sont dans une tranche d'âge critique, soit entre 45 et 64 ans. Parmi les 52 dirigeants qui " considèrent être trop tôt pour penser à la relève ", 1 dit vouloir se retirer de la direction dans 5 ans tandis que 8 veulent le faire dans 10 ans, ce qui, à notre avis, est représentatif d'un manque de sensibilisation à l'égard de la dynamique de la transmis- sion de leur entreprise, surtout s'ils envisagent une relève familiale.

\section{Conclusion}

Pour la majorité des propriétaires-dirigeants des PME manufacturières de la MRC de Drummond, assurer la relève de leur entreprise est important ou très important. Pourtant, plus de la moitié d'entre eux ne savent pas quand ils se retireront de la gouvernance de leur entreprise et plus des deux tiers ne savent pas quand ils procéderont au transfert de la propriété de cette même entreprise. Interrogés à cet effet, pour plusieurs, il serait trop tôt pour penser à la relève alors qu'ils seraient en âge d'y réfléchir. En bref, les propriétaires-dirigeants des PME manufacturières de la MRC de Drummond sont préoccupés par la problématique mais ne sauraient ni comment l'appréhender, ni comment procéder pour assurer la pérennité de leur entreprise. Par conséquent, nos résultats confirment la nécessité de la tenue d'activités de sensibilisation sur la relève, notamment auprès des propriétaires-dirigeants âgés de plus de 45 ans, ce qui représente une bonne partie d'entre eux. Toutefois, à la lumière de nos résultats, une mise en garde s'impose. Dans le but de rendre efficace une telle démarche de sensibilisation auprès des dirigeants des PME manufacturières de MRC de Drummond, les stratégies devront être adaptées pour prendre en compte les trois éléments suivants. D'abord, les trois quarts des propriétaires-dirigeants ayant participé à notre étude ont une formation académique de niveau secondaire ou collégial, ensuite, peu d'entre eux sont disponibles durant les heures d'affaires et enfin, comme leurs principaux confidents sont leurs conjoints et leurs enfants, il est clair pour nous que ces derniers devraient participer aux activités de sensibilisation sur la relève. Au reste, si nous revenons au principal objectif de cette étude, retenons que $41 \%$ des PME répondantes ont déjà de l'expérience en matière de transmission, tandis que $59 \%$ n'en ont pas. En bref, pour être efficaces, les intervenants socioéconomiques et les conseillers devront également penser à adapter leurs stratégies et leurs outils d'intervention, selon le «profil expérientiel » de l'entreprise. Finalement, considérant que les propriétaires-dirigeants que nous avons interrogés sont fortement préoccupés par la valeur de leur entreprise et par leur difficulté à trouver une relève compétente, sérieuse et intéressée; nous 
croyons qu'il serait pertinent de trouver des moyens leur permettant d'arrimer leurs attentes avec celles de candidats potentiels, permettant ainsi de conserver le dynamisme économique d'une région du Québec.

\section{Notes et références}

1 Voir le rapport de la FCEI (2003). Petites entreprises Grandes perspectives. www.fcei.ca

2 FCEI (2005). La relève : la clé de la réussite, Fédération canadienne de l'entreprise indépendante, www.fcei.ca.

3 OSEO (2005) «La transmission des petites et moyennes entreprises: l'expérience d'OSEO bdpme », www.OSEO.fr. OSEO ne représente pas un acronyme. Il s'agit du véritable nom de l'organisme né, en 2005, du rapprochement de l'ANVAR et de BDPME, autour d'une mission d'intérêt général, en appui des politiques régionales et nationales : accompagner et soutenir les PME-PMI - par des avances, prêts ou garanties - dans les phases les plus décisives de leur cycle de vie ( création, innovation, développement, transmission ). Il facilite l'accès des porteurs de projets et des entrepreneurs aux financements des partenaires bancaires et organismes de fonds propres (Information tirée de: http://www.oseo.fr/, saisie le 8 août 2006)

4 Par exemple, les résultats de la FCEI (2005) révèlent que $41 \%$ des dirigeants des PME canadiennes prévoient se retirer dans 5 ans alors que $30 \%$ ont un horizon temporel variant entre 6 et 10 ans.
5 Pour les cas de transmission familiale, voir les travaux de Hugron, P. (1992). L'entreprise familiale: modèle de réussite du processus successoral, monographie, publiée par: l'Institut de recherches politiques et les Presses HEC et de Cadieux, L. (2005). La succession dans les PME familiales : Proposition d'un modèle de réussite du processus de désengagement du prédécesseur, Revue Internationale PME, Vol. 18, no. 3-4, pages 31 à 50 . Pour les autres types de transmissions, voir le rapport de recherche d'OSEOpdpme (2005). La transmission des petites et moyennes entreprises: l'expérience d'OSEO bdpme. www.OSEO.fr; et les ouvrages de Mignon, S. (2001). Stratégie de pérennité d'entreprise, Vuibert, Paris, France; de Mossoux, J. (2004). Réussir la relève : transmission du patrimoine et du pouvoir. Éditions De Boeck Université et de Paturel, R. (2000). Reprise d'entreprise par une personne physique : une pratique de l'entrepreneuriat. in Verstraete, T. (dir.). Histoire d'entreprendre: Les réalités de l'entrepreneuriat, (p. 187-197). Cédex : Éditions EMS Management et Société. Pour le transfert de la propriété, voir Senbel, D. et St-Cyr, L. (2006). Analyse du transfert de propriété et de son financement au travers 20 cas de relève d'entreprises, cahier de recherche no. 06-01. HEC Montréal, Chaire de développement et de relève de la PME.

6 Voir Chrisman, J.-J., Chua, J.-H. et Sharma, P. (1998). Important attributes of successors in family businesses: an exploratory study, Family Business Review, XI(1), 19-34 et Sharma, P., Chrisman, J. et Chua, J. (2003). Succession planning as planned behaviour: some empirical results, Family Business Review, $X$ 


\section{Publicité}

«Doctorat en développement régional » 